\title{
MULTIPLIERS ON WEIGHTED HARDY SPACES OVER LOCALLY COMPACT VILENKIN GROUPS, I
}

\author{
C. W. ONNEWEER and T. S. QUEK
}

(Received 31 March 1989)

Communicated by I. Raeburn

\begin{abstract}
Let $G$ denote a locally compact Vilenkin group with dual group $\Gamma$. We give sufficient conditions for a function $\varphi \in L^{\infty}(\Gamma)$ to be a multiplier from the power-weighted Hardy space $H_{\alpha}^{p}(G)$ to itself or the corresponding power-weighted Lebesgue space $L_{\alpha}^{p}(G), 0<p \leq 1,-1<\alpha \leq 0$.

1980 Mathematics subject classification (Amer. Math. Soc.) (1985 Revision): primary 43 A 22; secondary 43 A 15,43 A 70.
\end{abstract}

\section{Introduction}

In a number of recent papers by $T$. Kitada [4], [5], [6] and by the present authors [7], [8], [9] various multiplier theorems for spaces of functions or distributions defined on locally compact Vilenkin groups were proved. The spaces considered in these papers were the $L^{p}$-spaces with power weights, $1 \leq p<\infty$, the $H^{p}$-spaces, $0<p<1$, and the power-weighted $H^{1}$ spaces. In the present paper we consider multipliers on power-weighted Hardy spaces $H_{\alpha}^{p}$, where $0<p \leq 1$ and $-1<\alpha \leq 0$. Our results are of two kinds: the first result, Theorem 4.5 , gives a sufficient condition for a function to be a multiplier from $H_{\alpha}^{p}$ to the corresponding power-weighted Lebesgue space $L_{\alpha}^{p}$, the second result, Theorem 4.7, deals with multipliers from $H_{\alpha}^{p}$ to $H_{\alpha}^{p}$. As a consequence of this last result we prove a multiplier theorem for $H_{\alpha}^{p}$ spaces, where the multiplier satisfies a Hörmander-type condition; see Theorem 4.15.

(C) 1990 Australian Mathematical Society 0263-6115/90\$A2.00+0.00 
Whereas some of the multiplier theorems in [4] have an analogue for function or distribution spaces on $\mathbb{R}^{n}$, for the multiplier theorems presented here no comparable version on $\mathbb{R}^{n}$ seems to be known.

We now give a brief outline of the paper. In the next section we introduce the necessary definitions and notation. In Section 3 we prove the equivalence of the maximal function characterization of the $H_{\alpha}^{p}$ spaces and their characterization in terms of weighted atoms. We also give an interpolation theorem for operators on $H^{p_{0}}$ spaces and $L^{p_{1}}$ spaces, $0<p_{0} \leq 1<p_{1}<\infty$. Section 4 is devoted to proofs of our main results and a brief discussion of the sharpness of the second of these results. We conclude that section, and the paper, by deriving the Hörmander-type multiplier theorem for the spaces $H_{\alpha}^{p}$.

\section{Definitions and notation}

Throughout this paper $G$ will denote a locally compact Abelian group containing a strictly decreasing sequence of open compact subgroups $\left(G_{n}\right)_{-\infty}^{\infty}$ such that

(i) $\sup \left\{\operatorname{order}\left(G_{n} / G_{n+1}\right): n \in \mathbb{Z}\right\}<\infty$,

(ii) $\bigcup_{-\infty}^{\infty} G_{n}=G$ and $\bigcap_{-\infty}^{\infty} G_{n}=\{0\}$.

Such groups are the locally compact analogue of the so-called Vilenkin groups which were first described by N. Ya. Vilenkin in 1947 [13]. Examples of such groups are given in [2, Section 4.1.2]. Additional examples are the additive group of the $p$-adic numbers and, more general, of a local field, see [11].

Let $\Gamma$ denote the dual group of $G$ and for each $n \in \mathbb{Z}$ let

$$
\Gamma_{n}=\left\{\gamma \in \Gamma: \gamma(x)=1 \text { for all } x \in G_{n}\right\} .
$$

We choose Haar measures $\mu$ on $G$ and $\lambda$ on $\Gamma$ so that $\mu\left(G_{0}\right)=\lambda\left(\Gamma_{0}\right)=1$. Then $\mu\left(G_{n}\right)=\left(\lambda\left(\Gamma_{n}\right)\right)^{-1}:=\left(m_{n}\right)^{-1}$ for each $n \in \mathbb{Z}$.

There exists a metric $d$ on $G \times G$ defined by $d(x, x)=0$ and $d(x, y)=$ $\left(m_{n}\right)^{-1}$ if $x-y \in G_{n} \backslash G_{n+1}$. Then the topology on $G$ determined by the metric $d$ coincides with the original topology on $G$. For $x \in G$ we set $\|x\|=d(x, 0)$. For each $\alpha \in \mathbb{R}$ we define the function $v_{\alpha}$ on $G$ by $v_{\alpha}(x)=$ $\|x\|^{\alpha}$; the corresponding measure $v_{\alpha} d \mu=\|x\|^{\alpha} d \mu$ will also be denoted by $d \mu_{\alpha}$. We mention here that a simple computation shows that $\mu_{\alpha}\left(G_{n}\right) \leq$ $C\left(m_{n}\right)^{-(\alpha+1)}$, provided $\alpha>-1$, and that $\mu_{\alpha}\left(x+G_{n}\right)=\left(m_{j}\right)^{-\alpha}\left(m_{n}\right)^{-1}$ if $x \in G_{j} \backslash G_{j+1}$ for some $j<n$. Here, like elsewhere, $C$ will denote a constant whose value may change from one occurrence to the next. The Lebesgue spaces on $G$ with respect to the measures $d \mu_{\alpha}$ will be denoted by $L_{\alpha}^{p}(G)$ or 
$L_{\alpha}^{p}$, and for $f \in L_{\alpha}^{p}, 0<p<\infty$ and $\alpha \in \mathbb{R}$ we set

$$
\|f\|_{p, \alpha}=\left(\int_{G}|f(x)|^{p} d \mu_{\alpha}(x)\right)^{1 / p}
$$

If $\alpha=0$ we write, as usual, $L^{p}$ and $\|f\|_{p}$ instead of $L_{0}^{p}$ and $\|f\|_{p, 0}$.

As a further generalization of the usual $L^{p}$ spaces we give here the definition of the Herz spaces on $G$. We shall use, both here and elsewhere, the notation $\chi_{A}$ for the characteristic function of a set $A$.

Definition 2.1. Let $0<p, q \leq \infty$ and $\alpha \in \mathbb{R}$. A measurable function $f: G \rightarrow \mathbb{C}$ belongs to the Herz space $K(\alpha, p, q ; G)=K(\alpha, p, q)$ if

$$
\|f\|_{K(\alpha, p, q)}:=\left(\sum_{l=-\infty}^{\infty}\left\|\left(m_{l}\right)^{-\alpha} f \chi_{G_{l} \backslash G_{l+1}}\right\|_{p}^{q}\right)^{1 / q}<\infty,
$$

with the usual modification if $q=\infty$.

It is easy to see that $K(\alpha / p, p, p)=L_{\alpha}^{p}$ for $\alpha \in \mathbb{R}$ and $0<p<\infty$.

We can also define a metric $\delta$ on $\Gamma \times \Gamma$ compatible with the topology on $\Gamma$. In this case we have $\|\gamma\|=\delta\left(\gamma, \gamma_{0}\right)=m_{n}$ if $\gamma \in \Gamma_{n+1} \backslash \Gamma_{n}$, where $\gamma_{0} \in \Gamma$ is defined by $\gamma_{0}(x)=1$ for all $x \in G$.

The symbols $\wedge$ and $\vee$ will be used to denote the Fourier transform and inverse Fourier transform, respectively. An easy computation shows that

and, hence

$$
\left(\chi_{G_{n}}\right)^{\wedge}=\left(\lambda\left(\Gamma_{n}\right)\right)^{-1} \chi_{\Gamma_{n}}=\left(m_{n}\right)^{-1} \chi_{\Gamma_{n}}
$$

$$
\left(\chi_{\Gamma_{n}}\right)^{\vee}=\left(\mu\left(G_{n}\right)\right)^{-1} \chi_{G_{n}}=m_{n} \chi_{G_{n}}:=\Delta_{n} .
$$

We now briefly review the definition of the spaces of test functions, $S(G)$, and distributions, $S^{\prime}(G)$; for more details, see [11]. A function $\varphi: G \rightarrow$ $\mathbb{C}$ belongs to $\varphi(G)$ if there exist integers $k, l$, depending on $\varphi$, so that $\operatorname{supp} \varphi \subset G_{k}$ and $\varphi$ is constant on the cosets of $G_{l}$ in $G$. A sequence $\left(\varphi_{n}\right)_{1}^{\infty}$ of functions in $S(G)$ converges to $\varphi \in S(G)$ if there exist $k, l \in \mathbb{Z}$ so that every $\varphi_{n}$ and $\varphi$ has support in $G_{k}$ and is constant on the cosets of $G_{l}$ in $G$ and if $\lim _{n \rightarrow \infty} \varphi_{n}(x)=\varphi(x)$ uniformly on $G$.

Next, $S^{\prime}(G)$ is the space of continuous linear functionals on $S(G)$. A sequence $\left(f_{n}\right)_{1}^{\infty}$ in $S^{\prime}(G)$ converges to $f \in S^{\prime}(G)$ if for all $\varphi \in S(G)$ we have $\lim _{n \rightarrow \infty}\left\langle f_{n}, \varphi\right\rangle=\langle f, \varphi\rangle$.

\section{Power-weighted Hardy spaces on $G$}

In [5] Kitada gave a definition for the Hardy spaces $H_{\alpha}^{l}(G)$ with respect to the weight functions $v_{\alpha}(x)=\|x\|^{\alpha}$, where $-1<\alpha \leq 0$. In the following 
we extend Kitada's definition. If $f \in S^{\prime}(G)$ we first define its regularization on $G \times \mathbb{Z}$ by $f(x, n)=f_{n}(x)=f * \Delta_{n}(x)$. Then $f_{n}$ is a function on $G$ which is constant on the cosets of $G_{n}$ in $G$. Moreover, $\lim _{n \rightarrow \infty} f_{n}=f$ in $S^{\prime}(G)$; see [11, Chapter IV]. For $f \in S^{\prime}(G)$ we define its maximal function $f^{*}$ by $f^{*}(x)=\sup _{n}\left|f * \Delta_{n}(x)\right|$.

Definition 3.1. Let $0<p<\infty$ and $\alpha \in \mathbb{R}$. The space $H_{\alpha}^{p}(G)=H_{\alpha}^{p}$ is the space of all $f \in S^{\prime}(G)$ for which $f^{*} \in L_{\alpha}^{p}$. We set

$$
\|f\|_{H_{\alpha}^{p}}=\left\|f^{*}\right\|_{p, \alpha}
$$

and we denote $H_{0}^{p}$ and $\|f\|_{H_{0}^{p}}$ by $H^{p}$ and $\|f\|_{H^{p}}$, respectively.

We now turn to the definition of the atomic Hardy spaces with power weight

Definition 3.2. Let $0<p \leq 1$ and $\alpha>-1$. A function $a: G \rightarrow \mathbb{C}$ is a $(p, \infty)_{\alpha}$ atom if there exists a set $I=x+G_{n}$ such that

(i) $\operatorname{supp} a \subset I$,

(ii) $\|a\|_{\infty} \leq\left(\mu_{\alpha}(I)\right)^{-1 / p}$,

(iii) $\int_{G} a(x) d \mu(x)=0$.

Clearly every $(p, \infty)_{\alpha}$ atom defines an element of $S^{\prime}(G)$. Moreover, an argument like in [1, page 611] shows that each $(p, \infty)_{\alpha}$ atom a belongs to $H_{\alpha}^{p}$ with $\|a\|_{H^{p}} \leq 1$.

Definition 3.3. Let $0<p \leq 1$ and $\alpha>-1$. The space $H_{\alpha}^{p, \infty}(G)=$ $H_{\alpha}^{p, \infty}$ is the space of all $f \in S^{\prime}(G)$ for which there exists a sequence $\left(\lambda_{i}\right)_{1}^{\infty} \in$ $l^{p}$ and a sequence of $(p, \infty)_{\alpha}$ atoms $\left(a_{i}\right)_{1}^{\infty}$ such that

$$
f=\sum_{i=1}^{\infty} \lambda_{i} a_{i} \text { in } s^{\prime}(G) \text {. }
$$

We set

$$
\|f\|_{H_{\alpha}^{p, \infty}}=\inf \left\{\left(\sum_{i=1}^{\infty}\left|\lambda_{i}\right|^{p}\right)^{1 / p}\right\},
$$

where the infimum is taken over all decompositions of $f$ of the form (3.4).

Theorem 3.5. Let $0<p \leq 1$ and $-1<\alpha \leq 0$. Then $H_{\alpha}^{p}=H_{\alpha}^{p, \infty}$ and the "norms" on these spaces are equivalent.

The proof of Theorem 3.5 will be preceded by a lemma.

LeMMA 3.6. Let $0<p \leq 1$ and $-1<\alpha \leq 0$. If $f \in H_{\alpha}^{p}$ then each $f_{n}=f * \Delta_{n}$ belongs to $H_{\alpha}^{p, \infty}$ and

with $C$ independent of $n \in \mathbb{Z}$.

$$
\left\|f_{n}\right\|_{H_{\alpha}^{p, \infty}} \leq C\|f\|_{H_{\alpha}^{p}}
$$


Proof. Let $f \in H_{\alpha}^{p}$ and for each $k \in \mathbb{Z}$ let

$$
\Omega_{k}=\left\{x \in G: f^{*}(x)>2^{k}\right\} .
$$

If $y \in \Omega_{k}$ then there exists an $N \in \mathbb{Z}$ so that $f_{N}(y)>2^{k}$ and this implies that $y+G_{N} \subset \Omega_{k}$. If $A(y)=\left\{n \in \mathbb{Z}: y+G_{n} \subset \Omega_{k}\right\}$, then $A(y)$ is bounded from below because $f^{*} \in L_{\alpha}^{p}$. Thus there exists an $\alpha(y) \in \mathbb{Z}$ so that $y+$ $G_{\alpha(y)} \subset \Omega_{k}$ and $y+G_{n} \not \subset \Omega_{k}$ for all $n<\alpha(y)$. We shall denote the at most countably many different sets $y+G_{\alpha(y)}$ with $y \in \Omega_{k}$ by $y_{k, i}+G_{\alpha(k, i)}:=I_{k, i}$. Then $\Omega_{k}=\bigcup_{i} I_{k, i}$ and $I_{k, i} \cap I_{k, j}=\varnothing$ for $i \neq j$.

Next, let $\tilde{I}_{k, i}=y_{k, i}+G_{\alpha(k, i)-1}$ and let $\tilde{\Omega}_{k}=\bigcup_{i} \tilde{I}_{k, i}$. If necessary we first rename the sets $\tilde{I}_{k, i}$ so that they are mutually disjoint.

Also, observe that for each $k \in \mathbb{Z}, \Omega_{k+1} \subset \Omega_{k}$ and, since $f \in H_{\alpha}^{p}$, $\mu_{\alpha}\left(\Omega_{k}\right)<\infty$ and $\mu_{\alpha}\left(\cap_{-\infty}^{\infty} \Omega_{k}\right)=0$, which implies that $\lim _{k \rightarrow \infty} \mu_{\alpha}\left(\Omega_{k}\right)=0$.

Next, for each function $f_{n}=f * \Delta_{n}$ and each $k \in \mathbb{Z}$, let

$$
\Omega_{k}^{n}=\left\{x \in G:\left|f_{n}(x)\right|>2^{k}\right\} .
$$

Then $\Omega_{k}^{n} \subset \Omega_{k}$.

For $k, n \in \mathbb{Z}$ we define the function $g_{k}^{n}: G \rightarrow \mathbb{C}$ by

$$
g_{k}^{n}(x)= \begin{cases}f_{n}(x) & \text { if } x \notin \tilde{\Omega}_{k}, \\ p_{k, i}^{n} & \text { if } x \in \tilde{I}_{k, i},\end{cases}
$$

where

$$
P_{k, i}^{n}=\left(\mu\left(\tilde{I}_{k, i}\right)\right)^{-1} \int_{\tilde{I}_{k, i}} f_{n}(x) d \mu(x) .
$$

We first show that for a.e. $x \in G$,

(i) $\lim _{k \rightarrow-\infty} g_{k}^{n}(x)=0$,

(ii) $\lim _{k \rightarrow \infty} g_{k}^{n}(x)=f_{n}(x)$.

To prove (i), consider $x \in \tilde{I}_{k, i}=y+G_{l}$, say. If $n \leq l$ then $f_{n}$ is constant on $y+G_{l}$ and, since $\tilde{I}_{k, i} \not \subset \Omega_{k}$ we see that $\left|f_{n}(x)\right| \leq 2^{k}$ on $\tilde{I}_{k, i}$ and this implies that $\left|P_{k, i}^{n}\right| \leq 2^{k}$. If $n>l$, then

$$
\mathrm{P}_{k, i}^{n}=\left(f * \Delta_{n}\right) * \Delta_{l}(y)=f * \Delta_{l}(y)=f_{l}(y),
$$

which again implies that $\left|P_{k, i}^{n}\right| \leq 2^{k}$. Therefore, we see that $\left|g_{k}^{n}(x)\right| \leq 2^{k}$ for all $x \in G$ and hence (i) holds.

To prove (ii), observe that $\mu_{\alpha}\left(\bigcap_{-\infty}^{\infty} \Omega_{k}\right)=0$ implies that $\mu\left(\bigcap_{-\infty}^{\infty} \Omega_{k}\right)=0$ and hence, $\mu\left(\cap_{-\infty}^{\infty} \tilde{\Omega}_{k}\right)=0$. This last equality immediately implies (ii). It 
follows from (i) and (ii) that for a.e. $x \in G$,

$$
f_{n}(x)=\sum_{k=-\infty}^{\infty}\left(g_{k+1}^{n}-g_{k}^{n}\right)(x)
$$

that is,

$$
f_{n}(x)=\sum_{k=-\infty}^{\infty} \sum_{i}\left(g_{k+1}^{n}-g_{k}^{n}\right)(x) \chi_{\tilde{I}_{k, i}}(x) .
$$

For each $k, i, n$ let

$$
b_{k, i}^{n}=\left(g_{k+1}^{n}-g_{k}^{n}\right) \chi_{I_{k, i}} .
$$

Then $\operatorname{supp} b_{k, i}^{n} \subset \tilde{I}_{k, i},\left\|b_{k, i}^{n}\right\|_{\infty} \leq 2^{k+2}$ and a routine calculation shows that

$$
\int_{G} b_{k, i}^{n}(x) d \mu(x)=0 .
$$

We now prove that

$$
f_{n}=\sum_{k=-\infty}^{\infty} \sum_{i} b_{k, i}^{n}
$$

with the series in (3.9) converging to $f_{n}$ in $S^{\prime}(G)$. To do so, take any $\varphi \in S(G)$ with, say, $\operatorname{supp} \varphi \subset G_{t}$ for some $t \in Z$. We need to prove that

$$
\lim _{\substack{n_{1} \rightarrow-\infty \\ n_{2}, n_{3} \rightarrow \infty}} \int_{G} \sum_{k=n_{1}}^{n_{2}} \sum_{i \leq n_{3}} b_{k, i}^{n}(x) \varphi(x) d \mu(x)=\int_{G} f_{n}(x) \varphi(x) d \mu(x) .
$$

We first prove three auxiliary results, (3.11), (3.12) and (3.13).

(3.11) There exists an $N_{1} \in-\mathbb{N}$ such that

$$
A:=\sum_{k=-\infty}^{N_{1}} \sum_{i}\left\|b_{k, i}^{n} \varphi\right\|_{1} \leq 1 \text {. }
$$

We have

$$
\begin{aligned}
A & \leq \sum_{k=-\infty}^{N_{1}}\left\|g_{k+1}^{n}-g_{k}^{n}\right\|_{\infty}\|\varphi\|_{1} \\
& \leq \sum_{k=-\infty}^{N_{1}} 2^{k+2}\|\varphi\|_{1} \leq 2^{N_{1}+3}\|\varphi\|_{1} \leq 1,
\end{aligned}
$$

for suitably chosen $N_{1} \in-\mathbb{N}$.

(3.12) There exists an $N_{2} \in \mathbb{N}$ so that for every $k>N_{2}$, every $i \in \mathbb{N}$ and $n \in \mathbb{Z}$,

$$
\left\langle b_{k, i}^{n}, \varphi\right\rangle=\int_{G} b_{k, i}^{n}(x) \varphi(x) d \mu(x)=0 .
$$


Since $\varphi \in S(G)$, there exists an $s \in \mathbb{Z}$ such that $\varphi$ is constant on the cosets of $G_{s}$ in $G$ but not on the cosets of $G_{s-1}$ (unless $\left.\varphi(x) \equiv 0\right)$. Hence there exist $x_{1}, \ldots, x_{r} \in G$ such that $x_{i}+G_{s} \cap x_{j}+G_{s}=\varnothing$ for $i \neq j$ and $\operatorname{supp} \varphi=\bigcup_{j=1}^{r} x_{j}+G_{s}$. Also, since $\lim _{k \rightarrow \infty} \mu_{\alpha}\left(\Omega_{k}\right)=0$, [7, Lemma 1(c)] implies that $\lim _{k \rightarrow \infty} \mu_{\alpha}\left(\tilde{\Omega}_{k}\right)=0$. Consequently, there exists an $N_{2} \in \mathbb{N}$ such that for all $k>N_{2}$ and all $i \in \mathbb{N}$ we have

$$
\mu_{\alpha}\left(\tilde{I}_{k, i}\right) \leq \mu_{\alpha}\left(\tilde{\Omega}_{k}\right) \leq \min \left\{\mu_{\alpha}\left(x_{j}+G_{s}\right): 1 \leq j \leq r\right\} .
$$

Because each $\tilde{I}_{k, i}$ is a coset of some subgroup $G_{l}$ of $G$ we see that for $k \geq N_{2}$ we have either $\tilde{I}_{k, i} \subset x_{j}+G_{s}$ for some $j$, or else $\tilde{I}_{k, i} \cap x_{j}+G_{s}=\varnothing$ for all $j, 1 \leq j \leq r$. In the latter case we have $\tilde{I}_{k, i} \cap \operatorname{supp} \varphi=\varnothing$ and hence $\left\langle b_{k, i}^{n}, \varphi\right\rangle=0$ for all $n \in \mathbb{Z}$; in case $\tilde{I}_{k, i} \subset x_{j}+G_{s}$ for some $j$ with $1 \leq j \leq r$, we again have $\left\langle b_{k, i}^{n}, \varphi\right\rangle=0$ for all $n \in \mathbb{Z}$, because (3.8) holds. This proves (3.12).

(3.13) With $N_{1}, N_{2}$ chosen so that (3.11) and (3.12) hold, there exists an $N_{3} \in \mathbb{N}$ so that

$$
B:=\sum_{k=N_{1}+1}^{N_{2}} \sum_{i \geq N_{3}}\left\|b_{k, i}^{n} \varphi\right\|_{1} \leq 1
$$

We have

$$
B \leq \sum_{k=N_{1}+1}^{N_{2}} \sum_{i \geq N_{3}} \int_{\tilde{I}_{k, i}}\left|\left(g_{k+1}^{n}-g_{k}^{n}\right)(x)\right||\varphi(x)| v_{-\alpha}(x) d \mu_{\alpha}(x) .
$$

Since $v_{-\alpha}(x) \leq\left(m_{t}\right)^{\alpha}$ for $\alpha \leq 0$ and $x \in G_{t}$, we see that

$$
B \leq\|\varphi\|_{\infty}\left(m_{t}\right)^{\alpha} \sum_{k=N_{1}+1}^{N_{2}} \sum_{i \geq N_{3}} 2^{k+2} \mu_{\alpha}\left(\tilde{I}_{k, i}\right)
$$

Now we observe that for every $k \in \mathbb{Z}$ there exists an $i_{k} \in \mathbb{N}$ so that

$$
\sum_{i \geq i_{k}} \mu_{\alpha}\left(\tilde{I}_{k, i}\right)<\left(2^{N_{2}+3}\|\varphi\|_{\infty}\left(m_{t}\right)^{\alpha}\right)^{-1}
$$

Let $N_{3}=\max \left\{i_{k}: N_{1}<k \leq N_{2}\right\}$. Then for this choice of $N_{3}$ we immediately obtain (3.13).

Applying (3.11), (3.12) and (3.13) it is easy to see that for every $n_{1} \in-\mathbb{N}$ and $n_{2}, n_{3} \in \mathbb{N}$,

$$
\sum_{k=n_{1}}^{n_{2}} \sum_{i \leq n_{3}} b_{k, i}^{n}(x) \varphi(x)
$$


is dominated pointwise on $G$ by an integrable function. Thus, in view of (3.7), the Lebesgue Dominated Convergence Theorem implies (3.10) and, therefore, (3.9).

Finally, let

$$
\lambda_{k, i}=2^{k+2}\left(\mu_{\alpha}\left(\tilde{I}_{k, i}\right)\right)^{1 / p} \quad \text { and } \quad a_{k, i}^{n}=\left(\lambda_{k, i}\right)^{-1} b_{k, i}^{n} .
$$

Then each $a_{k, i}^{n}$ is a $(p, \infty)_{\alpha}$ atom and

$$
f_{n}=\sum_{k, i} \lambda_{k, i} a_{k, i}^{n} \text { in } S^{\prime}(G) .
$$

Furthermore, a straightforward computation shows that

$$
\sum_{k, i}\left|\lambda_{k, i}\right|^{p} \leq C\left\|f_{n}^{*}\right\|_{p, \alpha}^{p} \leq C\left\|f^{*}\right\|_{p, \alpha}^{p}=C\|f\|_{H_{\alpha}^{p}}^{p}
$$

This completes the proof of Lemma 3.6.

Proof of Theorem 3.5. Take any $f \in H_{\alpha}^{p}$. Using the same notation as in the proof of Lemma 3.6, we see from the definition of the $(p, \infty)_{\alpha}$ atoms $a_{k, i}^{n}$ that

$$
\sup _{n \in \mathbb{N}}\left\|a_{0,1}^{n}\right\|_{\infty} \leq\left(\mu_{\alpha}\left(\tilde{I}_{0,1}\right)\right)^{-1 / p} .
$$

Thus the Banach-Alaoglu theorem implies the existence of a subsequence $\left(a_{0,1}^{n_{\nu(0,1)}}\right)$ of $\left(a_{0,1}^{n}\right)$ so that this subsequence converges in the weak ${ }^{*}$ topology of $L^{\infty}(G)$ to, say, $a_{0,1} \in L^{\infty}(G)$. Clearly, $a_{0,1}$ is a $(p, \infty)_{\alpha}$ atom with $\operatorname{supp} a_{0,1} \subset \tilde{I}_{0,1}$. Next, since

$$
\sup _{n_{\nu(0,1)}}\left\|a_{1,1}^{n_{\nu(0,1)}}\right\|_{\infty} \leq\left(\mu_{\alpha}\left(\tilde{I}_{1,1}\right)\right)^{-1 / p}
$$

a second application of the Banach-Alaoglu theorem yields a subsequence $\left(a_{1,1}^{n_{\nu(1)}}\right)$ of $\left(a_{1,1}^{n_{\nu(0,1)}}\right)$ and a $(p, \infty)_{\alpha}$ atom $a_{1,1}$ with supp $a_{1,1} \subset \tilde{I}_{1,1}$ so that the subsequence converges weak ${ }^{*}$ in $L^{\infty}(G)$ to $a_{1,1}$. Arranging the pairs of subscripts $(k, i)$ with $k \in \mathbb{Z}$ and $i \in \mathbb{N}$ in a sequence we can repeat the process described above for each $(k, i)$. By the usual diagonalization method we obtain a sequence $\left(n_{\nu}\right)$ and a sequence of $(p, \infty)_{\alpha}$ atoms $a_{k, i}$ with $\operatorname{supp} a_{k, i} \subset \tilde{I}_{k, i}$ so that for all $(k, i)$ we have

$$
\lim _{\nu \rightarrow \infty} a_{k, i}^{n_{\nu}}=a_{k, i} \quad \text { weak }^{*} \text { in } L^{\infty} \text {. }
$$

We shall prove that

$$
f=\sum_{k=-\infty}^{\infty} \sum_{i} \lambda_{k, i} a_{k, i} \text { in } S^{\prime}(G) .
$$


To do so, take any $\varphi \in S(G)$ and assume, like in Lemma 3.6, that supp $\varphi \subset$ $G_{t}$. Let $\varepsilon>0$ be given. We first derive three auxiliary inequalities, (3.16), (3.17) and (3.18).

(3.16) There exists an $M_{1} \in-\mathbb{N}$ so that for all $n \in \mathbb{Z}$ we have

(i) $\sum_{k=-\infty}^{M_{1}} \sum_{i}\left|\left\langle\lambda_{k, i} a_{k, i}^{n}, \varphi\right\rangle\right|<\frac{\varepsilon}{12}$,

(ii) $\sum_{k=-\infty}^{M_{1}} \sum_{i}\left|\left\langle\lambda_{k, i} a_{k, i}, \varphi\right\rangle\right|<\frac{\varepsilon}{12}$.

The proof of (3.16) is virtually the same as the proof of (3.11).

(3.17) There exists an $M_{2} \in \mathbb{N}$ so that for all $k>M_{2}$, every $i \in \mathbb{N}$ and $n \in \mathbb{Z}$ we have

(i) $\left\langle\lambda_{k, i} a_{k, i}^{n}, \varphi\right\rangle=0$,

(ii) $\left\langle\lambda_{k, i} a_{k, i}, \varphi\right\rangle=0$.

This is essentially a restatement of (3.12) with $M_{2}=N_{2}$.

(3.18) With $M_{1}, M_{2}$ chosen as in (3.16) and (3.17), there exists an $M_{3} \in$ $\mathbb{N}$ and an $n_{\nu_{1}} \in\left(n_{\nu}\right)_{\nu=1}^{\infty}$ so that for all $n_{\nu} \geq n_{\nu_{1}}$ we have

$$
\sum_{k=M_{1}+1}^{M_{2}} \sum_{i}\left|\lambda_{k, i}\right|\left|\left\langle a_{k, i}^{n_{\nu}}-a_{k, i}, \varphi\right\rangle\right|<\frac{\varepsilon}{6} .
$$

To prove (3.18), we observe that for each $k \in \mathbb{Z}$ the sets $\tilde{I}_{k, i}$ are mutually disjoint so that at most $r$ of the sets $\tilde{I}_{k, i}$ will contain at least one of the sets $x_{j}+G_{s}$, with $x_{j}+G_{s}$ as defined in the proof of (3.12). Let

$$
\tilde{i}_{k}=\max \left\{i: x_{j}+G_{s} \subset \tilde{I}_{k, i} \text { for some } j \text { with } 1 \leq j \leq r\right\},
$$

and let

$$
M_{3}=\max \left\{\tilde{i}_{k}: M_{1}<k \leq M_{2}\right\} .
$$

Clearly, if $M_{1}<k \leq M_{2}, i>M_{3}$ and $n \in \mathbb{Z}$, then $\left\langle a_{k, i}^{n}, \varphi\right\rangle=\left\langle a_{k, i}, \varphi\right\rangle=$ 0 . Furthermore, in view of (3.14) there exists an $n_{\nu_{1}}$ so that for all $n_{\nu} \geq n_{\nu_{1}}$ we have

$$
\begin{aligned}
& \sum_{k=M_{1}+1}^{M_{2}} \sum_{i}\left|\lambda_{k, i}\right|\left|\left\langle a_{k, i}^{n_{\nu}}-a_{k, i}, \varphi\right\rangle\right| \\
& \quad=\sum_{k=M_{1}+1}^{M_{2}} \sum_{i \leq M_{3}}\left|\lambda_{k, i}\right|\left|\left\langle a_{k, i}^{n_{\nu}}-a_{k, i}, \varphi\right\rangle\right|<\frac{\varepsilon}{6},
\end{aligned}
$$

which proves (3.18).

Now we observe that since $\lim _{n \rightarrow \infty} f_{n}=f$ in $S^{\prime}(G)$, there exists an $n_{\nu_{2}} \geq n_{\nu_{1}}$ so that

$$
\left|\left\langle f_{n_{\nu_{2}}}-f, \varphi\right\rangle\right|<\frac{\varepsilon}{3}
$$


In the proof of Lemma 3.6 we saw that there exist $N_{1} \in-\mathbb{N}$ and $N_{2}, N_{3} \in \mathbb{N}$, with $N_{1}, N_{2}, N_{3}$ depending on $n_{\nu_{2}}$, so that if $n_{1} \leq N_{1}, n_{2} \geq N_{2}$ and $n_{3} \geq N_{3}$ then

$$
\left|\sum_{k=n_{1}}^{n_{2}} \sum_{i \leq n_{3}}\left\langle\lambda_{k, i} a_{k, i}^{n_{\nu_{2}}}-f_{n_{\nu_{2}}}, \varphi\right\rangle\right|<\frac{\varepsilon}{3} .
$$

Consequently, if $l_{1} \leq \min \left\{M_{1}, N_{1}\right\}, l_{2} \geq N_{2}$ and $l_{3} \geq \max \left\{M_{3}, N_{3}\right\}$ then

$$
\begin{aligned}
& \left|\left\langle f-\sum_{k=l_{1}}^{l_{2}} \sum_{i \leq l_{3}} \lambda_{k, i} a_{k, i}, \varphi\right\rangle\right| \\
& =\left|\left\langle f-\sum_{k=l_{1}}^{N_{2}} \sum_{i \leq l_{3}} \lambda_{k, i} a_{k, i}, \varphi\right\rangle\right| \\
& \leq\left|\left\langle f-f_{n_{\nu_{2}}}, \varphi\right\rangle\right|+\left|\left\langle f_{n_{\nu_{2}}}-\sum_{k=l_{1}}^{N_{2}} \sum_{i \leq l_{3}} \lambda_{k, i} a_{k, i}^{n_{\nu_{2}}}, \varphi\right\rangle\right| \\
& +\sum_{k=M_{1}+1}^{N_{2}} \sum_{i \leq I_{3}}\left|\lambda_{k, i}\right|\left\langle a_{k, i}^{n_{\nu_{2}}}-a_{k, i}, \varphi\right\rangle \mid \\
& +\sum_{k=l_{1}}^{M_{1}} \sum_{i \leq l_{3}}\left|\left\langle\lambda_{k, i} a_{k, i}^{n_{\nu_{2}}}, \varphi\right\rangle\right|+\sum_{k=l_{1}}^{M_{1}} \sum_{i \leq l_{3}}\left|\left\langle\lambda_{k, i} a_{k, i}, \varphi\right\rangle\right| \\
& \leq \varepsilon / 3+\varepsilon / 3+\varepsilon / 6+\varepsilon / 12+\varepsilon / 12=\varepsilon .
\end{aligned}
$$

This proves (3.15). In the proof of Lemma 3.6 we saw that

$$
\sum_{k, i}\left|\lambda_{k, i}\right|^{p} \leq C\|f\|_{H_{\alpha}^{p}}^{p}
$$

Therefore, $f \in H_{\alpha}^{p, \infty}$ and

$$
\|f\|_{H_{\alpha}^{p, \infty}} \leq C\|f\|_{H_{\alpha}^{p}} .
$$

To prove the converse, take any $f \in H_{\alpha}^{p, \infty}$. Then $f=\sum_{k} \lambda_{k} a_{k}$ in $S^{\prime}(G)$, where $\left(\lambda_{k}\right) \in l^{p}$ and each $a_{k}$ is a $(p, \infty)_{\alpha}$ atom so that $\left\|a_{k}^{*}\right\|_{p, \alpha} \leq 1$. Consequently,

$$
\left|f^{*}(x)\right|^{p} \leq \sum_{k}\left|\lambda_{k}\right|^{p}\left|a_{k}^{*}(x)\right|^{p}
$$

and this implies that $\left\|f^{*}\right\|_{p, \alpha}^{p} \leq \sum_{k}\left|\lambda_{k}\right|^{p}$, that is, $f \in H_{\alpha}^{p}$ and

$$
\|f\|_{H_{\alpha}^{p}} \leq\|f\|_{H_{\alpha}^{p, \infty}}
$$

This completes the proof of Theorem 3.5.

We mention here the following corollary whose simple proof will be omitted. 
Corollary 3.21. For each $q$ with $1 \leq q<\infty$ we have $L^{q} \cap H_{\alpha}^{p}$ is dense in $H_{\alpha}^{p}$.

The last theorem of this section is an interpolation theorem for operators on $H^{p}$ and $L^{p}$ spaces. The theorem is a version for locally compact Vilenkin groups of [3, Theorems III.6.4 and 6.5] or [1, Theorem D], where also the precise definitions of some of the concepts used here can be found.

THEOREM 3.22. Let $0<p_{0} \leq 1<p_{1}<\infty$. Suppose $T$ is a sublinear operator of weak type $\left(H^{p_{0}}, p_{0}\right)$ on $H^{p_{0}}$ and of weak type $\left(p_{1}, p_{1}\right)$ on $L^{p_{1}}$. Then $T$ is bounded from $H^{p}$ to $L^{p}$ for $p_{0}<p \leq 1$ and $T$ is bounded form $L^{p}$ to $L^{p}$ for $1<p<p_{1}$.

Proof (Outline). Let $f \in L^{p}$ with $1<p<p_{1}$ and choose $q$ so that $1<q<p$. For $t>0$ let

$$
E_{t}=\left\{x: M_{q}(|f|)(x)=\left(|f|^{q}\right)^{*}(x)>t^{q}\right\} .
$$

As in the proof of Lemma 3.6 we can express $E_{t}$ as a disjoint union of maximal cosets of certain subgroups $G_{n}$ of $G$, say $E_{t}=\bigcup_{j} I_{j}$.

Define $g_{t}: G \rightarrow \mathbb{C}$ by

$$
g_{t}(x)= \begin{cases}f(x) & \text { if } x \notin E_{t}, \\ \left(\mu\left(I_{j}\right)\right)^{-1} \int_{I_{j}} f(x) d \mu(x) & \text { if } x \in I_{j},\end{cases}
$$

and define $b_{t}: G \rightarrow \mathbb{C}$ by $b_{t}(x)=f(x)-g_{t}(x)$. Then

$$
b_{t}(x)=\sum_{j}\left(f-g_{t}\right)(x) \chi_{I_{j}}(x)=\sum_{j} b_{j}(x) .
$$

We have

$$
\left(\left(\mu\left(I_{j}\right)\right)^{-1} \int_{I_{j}}\left|b_{j}(x)\right|^{q} d \mu(x)\right)^{1 / q} \leq C t,
$$

and if we set

$$
a_{j}(x)=\left(C t\left(\mu\left(I_{j}\right)\right)^{1 / p_{0}}\right)^{-1} b_{j}(x),
$$

then each $a_{j}$ is a $\left(p_{0}, q\right)$ atom and

$$
b_{t}(x)=\sum_{j} C t\left(\mu\left(I_{j}\right)\right)^{1 / p} a_{j}(x) .
$$

Thus $b_{t} \in H^{p_{0}, q}$ and $\left\|b_{t}\right\|_{H^{p_{0}}} \leq C t\left(\mu\left(E_{t}\right)\right)^{1 / p}$. Moreover, $\left|g_{t}(x)\right| \leq C t$ for $x \in E_{t}$, and for $x \notin E_{t}$ we have $|f(x)| \leq M(|f|)(x) \leq\left\{M_{q}(|f|)(x)\right\}^{1 / q} \leq t$. 
Consequently,

$$
\begin{aligned}
& \int_{G}\left|g_{t}(x)\right|^{p_{1}} d \mu(x)=\int_{G \backslash E_{t}}\left|g_{t}(x)\right|^{p_{1}} d \mu(x)+\int_{E_{t}}\left|g_{t}(x)\right|^{p_{1}} d \mu(x) \\
& \quad \leq \int_{|f| \leq t}|f(x)|^{p_{1}} d \mu(x)+(C t)^{p_{1}} \mu\left(E_{t}\right) \\
& \quad \leq C t^{p_{1}-p}\|f\|_{p}^{p}
\end{aligned}
$$

that is, $g_{t} \in L^{p_{1}}$ for every $t>0$. The rest of the proof is virtually the same as the proof of [3, Theorems III.6.4 and 6.5] and will be omitted.

\section{Multipliers on $H_{\alpha}^{p}(G)$}

As mentioned in the introduction, in this section we shall present our multiplier theorems for the spaces $H_{\alpha}^{p}$. Throughout this section, if $\varphi \in$ $L^{\infty}(\Gamma)$ and if $k \in \mathbb{Z}$ we let $\varphi_{k}=\varphi \chi_{\Gamma_{k}}$ and $\varphi^{k}=\varphi_{k+1}-\varphi_{k}$. We begin with a definition which extends a definition given by Kitada in [5].

Definition 4.1. Let $0<p \leq 1$ and $-1<\alpha \leq 0$. Let $X$ denote $H_{\alpha}^{p}$ and let $Y$ denote $H_{\alpha}^{p}$ or $L_{\alpha}^{p}$. A function $\varphi \in L^{\infty}(\Gamma)$ is a multiplier from $X$ to $Y(\varphi \in \mathcal{M}(X, Y)$ or $\varphi \in \mathcal{M}(X)$ in case $X=Y)$ if there exists a constant $C>0$ so that for all $f \in X \cap L^{2}$ we have $(\varphi \hat{f})^{\vee} \in Y$ and $\left\|(\varphi \hat{f})^{\vee}\right\|_{Y} \leq C\|f\|_{X}$.

REMARK 4.2. In order to prove that $\varphi \in \mathcal{M}(X, Y)$ it is sufficient to prove that there exists a $C>0$ so that for every $(p, \infty)_{\alpha}$ atom $a$ and for every $k \in \mathbb{Z}$ we have $\left\|\left(\varphi_{k}\right)^{\vee} * a\right\|_{Y}=\left\|\left(\varphi_{k} \hat{a}\right)^{\vee}\right\|_{Y} \leq C$. To see this, take any $(p, \infty)_{\alpha}$ atom $a$ and let $\hat{a}_{k}=\hat{a} \chi_{\Gamma_{k}}$. Then $\lim _{k \rightarrow \infty} \hat{a}_{k}=\hat{a}$ in $L^{2}(\Gamma)$. Consequently,

$$
\lim _{k \rightarrow \infty} \varphi_{k} \hat{a}=\lim _{k \rightarrow \infty} \varphi \hat{a}_{k}=\varphi \hat{a} \quad \text { in } L^{2}(\Gamma)
$$

and hence,

$$
\lim _{k \rightarrow \infty}\left(\varphi_{k} \hat{a}\right)^{\vee}=(\varphi \hat{a})^{\vee} \quad \text { in } L^{2}(G) .
$$

Now we distinguish two cases.

(i) Let $Y=L_{\alpha}^{p}$. Then (4.3) implies the existence of a subsequence $\left(k_{i}\right)$ so that

$$
\lim _{i \rightarrow \infty}\left(\varphi_{k_{i}} \hat{a}\right)^{\vee}(x)=(\varphi \hat{a})^{\vee}(x) \text { for a.e. } x \in G .
$$

Thus, Fatou's Lemma implies that

$$
\left\|(\varphi \hat{a})^{\vee}\right\|_{p, \alpha} \leq \liminf \left\|\left(\varphi_{k_{i}} \hat{a}\right)^{\vee}\right\|_{p, \alpha} \leq C .
$$


From this inequality we easily derive that $\varphi \in \mathcal{M}(X, Y)$.

(ii) Let $Y=X=H_{\alpha}^{p}$. Then (4.3) implies that

$$
\lim _{k \rightarrow \infty}\left(\varphi_{k} \hat{a}\right)^{\vee}=(\varphi \hat{a})^{\vee} \text { in } S^{\prime}(G) \text {. }
$$

Now a simple argument shows that for every $x \in G$,

$$
\left(\left((\varphi \hat{a})^{\vee}\right)^{*}(x)\right)^{p} \leq \liminf \left(\left(\left(\varphi_{k} \hat{a}\right)^{\vee}\right)^{*}(x)\right)^{p}
$$

and an application of Fatou's Lemma shows that

$$
\left\|(\varphi \hat{a})^{\vee}\right\|_{H_{\alpha}^{p}} \leq \liminf \left\|\left(\varphi_{k} \hat{a}\right)^{\vee}\right\|_{H_{\alpha}^{p}} \leq C
$$

and this inequality immediately implies that $\varphi \in \mathcal{M}(X)$.

We now turn to the discussion of our multiplier theorems for the spaces $H_{\alpha}^{p}$. Our first result deals with multipliers from the spaces $H_{\alpha}^{p}$ to the corresponding spaces $L_{\alpha}^{p}$. We start with a lemma in which we consider the case $\alpha=0$.

LEMMA 4.4. Let $\varphi \in L^{\infty}(\Gamma)$ and let $0<p \leq 1$. If

$$
\sup _{k}\left(m_{k}\right)^{1 / p-1}\left\|\left(\varphi_{k}\right)^{\vee}\right\|_{K(1 / p-1 / r, r, p)}<\infty
$$

for some $r$ with $p<r<\infty$ then

(i) $\varphi \in \mathcal{M}\left(H^{s}, L^{s}\right)$ for $p \leq s \leq 1$ and

(ii) $\varphi \in \mathcal{M}\left(L^{s}\right)$ for $1<s<\infty$.

Proof. Let $a$ be a $(p, \infty)$ atom with supp $a \subset I=x_{0}+G_{n}$ for some $x_{0} \in G$ and $n \in \mathbb{Z}$. For every $k \in \mathbb{Z}$ we have

$$
\begin{aligned}
\left\|\left(\varphi_{k} \hat{a}\right)^{\vee}\right\|_{p}^{p} & =\left\|\left(\varphi_{k}\right)^{\vee} * a\right\|_{p}^{p} \\
& =\left\|\left(\left(\varphi_{k}\right)^{\vee} * a\right) \chi_{I}\right\|_{p}^{p}+\left\|\left(\left(\varphi_{k}\right)^{\vee} * a\right) \chi_{G \backslash I}\right\|_{p}^{p}:=A+B .
\end{aligned}
$$

Applying Hölder's inequality we see that

$$
\begin{aligned}
A & \leq\left(\int_{I}\left|\left(\varphi_{k}\right)^{\vee} * a(x)\right|^{2} d \mu(x)\right)^{p / 2} \cdot(\mu(I))^{1-p / 2} \\
& \leq C\left\|\left(\varphi_{k}\right)^{\vee} * a\right\|_{2}^{p} \cdot\left(m_{n}\right)^{-(1-p / 2)} \\
& \leq C\left\|\varphi_{k}\right\|_{\infty}^{p}\|a\|_{2}^{p} \cdot\left(m_{n}\right)^{-(1-p / 2)} \\
& \leq C\|\varphi\|_{\infty}^{p},
\end{aligned}
$$

because $a$ is a $(p, \infty)$ atom. 
For $B$ we have

$$
\begin{aligned}
B & =\int_{G \backslash I}\left|\int_{G}\left(\varphi_{k}\right)^{\vee}(t) a(x-t) d \mu(t)\right|^{p} d \mu(x) \\
& \leq\|a\|_{\infty}^{p} \int_{G \backslash I}\left(\int_{x-I}\left|\left(\varphi_{k}\right)^{\vee}(t)\right| d \mu(t)\right)^{p} d \mu(x) .
\end{aligned}
$$

Since $\varphi_{k}(\gamma)=0$ for $\gamma \in \Gamma \backslash \Gamma_{k},\left(\varphi_{k}\right)^{\vee}$ is constant on the cosets of $G_{k}$. Thus, if $\left(x_{i}+G_{k}\right)_{i=0}^{\infty}$ represent the different cosets of $G_{k}$ in $G$, then

$$
\left(\varphi_{k}\right)^{\vee}(t)=\sum_{i=0}^{\infty}\left(\varphi_{k}\right)^{\vee}\left(x_{i}\right) \chi_{x_{i}+G_{k}}(t),
$$

so

$$
\begin{aligned}
& \left(\int_{x-I}\left|\left(\varphi_{k}\right)^{\vee}(t)\right| d \mu(t)\right)^{p}=\left(\sum_{\left\{i: x_{i} \in x-I\right\}}\left|\left(\varphi_{k}\right)^{\vee}\left(x_{i}\right)\right|\left(m_{k}\right)^{-1}\right)^{p} \\
& \quad \leq \sum_{\left\{i: x_{i} \in x-I\right\}}\left|\left(\varphi_{k}\right)^{\vee}\left(x_{i}\right)\right|^{p}\left(m_{k}\right)^{-p} \\
& \quad=\left(m_{k}\right)^{-(p-1)} \int_{x-I}\left|\left(\varphi_{k}\right)^{\vee}(t)\right|^{p} d \mu(t) .
\end{aligned}
$$

Therefore,

$$
\begin{aligned}
B & \leq\|a\|_{\infty}^{p} \cdot\left(m_{k}\right)^{1-p} \int_{G \backslash I} \int_{I}\left|\left(\varphi_{k}\right)^{\vee}(x-t)\right|^{p} d \mu(t) d \mu(x) \\
& =\|a\|_{\infty}^{p} \cdot\left(m_{k}\right)^{1-p} \int_{G_{n}} \int_{G \backslash G_{n}}\left|\left(\varphi_{k}\right)^{\vee}(y-u)\right|^{p} d \mu(y) d \mu(u) .
\end{aligned}
$$

Next we observe that for each $u \in G_{n}$,

$$
\begin{aligned}
\int_{G \backslash G_{n}}\left|\left(\varphi_{k}\right)^{\vee}(y-u)\right|^{p} d \mu(y)=\int_{G \backslash G_{n}}\left|\left(\varphi_{k}\right)^{\vee}(y)\right|^{p} d \mu(y) \\
\quad=\sum_{j=-\infty}^{n-1} \int_{G_{j} \backslash G_{j+1}}\left|\left(\varphi_{k}\right)^{\vee}(y)\right|^{p} d \mu(y) \\
\leq \sum_{j=-\infty}^{n-1}\left(\int_{G_{j} \backslash G_{j+1}}\left|\left(\varphi_{k}\right)^{\vee}(y)\right|^{r} d \mu(y)\right)^{p / r} \cdot\left(\mu\left(G_{j} \backslash G_{j+1}\right)\right)^{1-p / r} \\
\leq C \sum_{j=-\infty}^{n-1}\left(\left(m_{j}\right)^{-(1 / p-1 / r)}\left\|\left(\varphi_{k}\right)^{\vee} \chi_{G_{j} \backslash G_{j+1}}\right\|_{r}\right)^{p} \\
\leq C\left\|\left(\varphi_{k}\right)^{\vee}\right\|_{K(1 / p-1 / r, r, p)}^{p} .
\end{aligned}
$$


Since $\|a\|_{\infty} \leq(\mu(I))^{-1 / p} \leq C\left(m_{n}\right)^{1 / p}$, we see that

$$
B \leq C m_{n}\left(m_{k}\right)^{1-p}\left(m_{n}\right)^{-1}\left(m_{k}\right)^{p-1}=C .
$$

Consequently, $\varphi \in \mathcal{M}\left(H^{p}, L^{p}\right)$. Since $\varphi \in L^{\infty}(\Gamma)$, we have $\varphi \in \mathcal{M}\left(L^{2}\right)$. Thus, an application of Theorem 3.22 and a duality argument complete the proof of the lemma.

THEOREM 4.5. Let $\varphi \in L^{\infty}(\Gamma)$ and let $0<p \leq 1$. If

$$
\sup _{k}\left(m_{k}\right)^{1 / p-1}\left\|\left(\varphi_{k}\right)^{\vee}\right\|_{K(1 / p-1 / r, r, p)}<\infty
$$

for some $r$ with $p<r<\infty$, then $\varphi \in \mathcal{M}\left(H_{\alpha}^{p}, L_{\alpha}^{p}\right)$ for $-1+p / r<\alpha \leq 0$.

Proof. Let $a$ be a $(p, \infty)_{\alpha}$ atom. We shall distinguish two cases, depending on supp $a$. First assume supp $a \subset I=x_{0}+G_{n}$ with $x_{0} \notin G_{n}$. Then $x_{0} \in G_{j} \backslash G_{j+1}$ for some $j<n$ and $\mu_{\alpha}(I)=\left(m_{j}\right)^{-\alpha}\left(m_{n}\right)^{-1}$, so that $\|a\|_{\infty} \leq\left(\left(m_{j}\right)^{\alpha} m_{n}\right)^{1 / p}$. For each $k \in \mathbb{Z}$ we have

$$
\begin{aligned}
\left\|\left(\varphi_{k} \hat{a}\right)^{\vee}\right\|_{p, \alpha}^{p} & =\left\|\left(\varphi_{k}\right)^{\vee} * a\right\|_{p, \alpha}^{p} \\
& =\left\|\left(\left(\varphi_{k}\right)^{\vee} * a\right) \chi_{I}\right\|_{p, \alpha}^{p}+\left\|\left(\left(\varphi_{k}\right)^{\vee} * a\right) \chi_{G \backslash I}\right\|_{p, \alpha}^{p}:=A+B,
\end{aligned}
$$

where

$$
\begin{aligned}
A & =\int_{I}\left|\left(\varphi_{k}\right)^{\vee} * a(x)\right|^{p} v_{\alpha}(x) d \mu(x) \\
& \leq\left(m_{j}\right)^{-\alpha}\left(\int_{I}\left|\left(\varphi_{k}\right)^{\vee} * a(x)\right|^{2} d \mu(x)\right)^{p / 2} \cdot(\mu(I))^{1-p / 2} \\
& \leq\left(m_{j}\right)^{-\alpha}\left(m_{n}\right)^{-(1-p / 2)}\left\|\left(\varphi_{k}\right)^{\vee} * a\right\|_{2}^{p} \\
& \leq\left(m_{j}\right)^{-\alpha}\left(m_{n}\right)^{-(1-p / 2)}\|\varphi\|_{\infty}^{p}\|a\|_{2}^{p} \leq\|\varphi\|_{\infty}^{p} .
\end{aligned}
$$

To estimate $B$ we observe that, as in the proof of Lemma 4.4,

$$
\begin{aligned}
B & =\int_{G \backslash I}\left|\int_{G}\left(\varphi_{k}\right)^{\vee}(x-t) a(t) d \mu(t)\right|^{p} d \mu_{\alpha}(x) \\
& \leq\|a\|_{\infty}^{p} \int_{G \backslash I}\left(\int_{x-I}\left|\left(\varphi_{k}\right)^{\vee}(t)\right| d \mu(t)\right)^{p} d \mu_{\alpha}(x) \\
& \leq\|a\|_{\infty}^{p} \cdot\left(m_{k}\right)^{1-p} \int_{G \backslash I} \int_{I}\left|\left(\varphi_{k}\right)^{\vee}(x-t)\right|^{p} d \mu(t) d \mu_{\alpha}(x) \\
& \leq\|a\|_{\infty}^{p} \cdot\left(m_{k}\right)^{1-p} \int_{G_{n}} \int_{G \backslash G_{n}}\left|\left(\varphi_{k}\right)^{\vee}(y-u)\right|^{p} v_{\alpha}\left(x_{0}+y\right) d \mu(y) d \mu(u) .
\end{aligned}
$$


We now estimate the inner integral, first writing it as a sum of three integrals

$$
\begin{aligned}
\int_{G \backslash G_{n}} \cdots d \mu(y) & =\int_{G \backslash G_{j}} \cdots+\int_{G_{j} \backslash G_{j+1}} \cdots+\int_{G_{j+1} \backslash G_{n}} \cdots d \mu(y) \\
& :=B_{1}+B_{2}+B_{3} .
\end{aligned}
$$

For $x_{0} \in G_{j} \backslash G_{j+1}$ and $y \notin G_{j}$ we have $x_{0}+y \notin G_{j}$, so that $v_{\alpha}\left(x_{0}+y\right) \leq$ $\left(m_{j}\right)^{-\alpha}$. Therefore, if $u \in G_{n}$ we obtain, as in the proof of Lemma 4.4,

$$
\begin{aligned}
B_{1} & \leq\left(m_{j}\right)^{-\alpha} \int_{G \backslash G_{n}}\left|\left(\varphi_{k}\right)^{\vee}(y-u)\right|^{p} d \mu(y) \\
& \leq C\left(m_{j}\right)^{-\alpha}\left\|\left(\varphi_{k}\right)^{\vee}\right\|_{K(1 / p-1 / r, r, p)}^{p} \leq C\left(m_{j}\right)^{-\alpha}\left(m_{k}\right)^{p-1} .
\end{aligned}
$$

For $x_{0} \in G_{j} \backslash G_{j+1}$ and $y \in G_{j+1} \backslash G_{n}$ we have $x_{0}+y \in G_{j} \backslash G_{j+1}$ and hence, $v_{\alpha}\left(x_{0}+y\right)=\left(m_{j}\right)^{-\alpha}$. Therefore, if $u \in G_{n}$ then

$$
B_{3} \leq\left(m_{j}\right)^{-\alpha} \int_{G \backslash G_{n}}\left|\left(\varphi_{k}\right)^{\vee}(y-u)\right|^{p} d \mu(y) \leq C\left(m_{j}\right)^{-\alpha}\left(m_{k}\right)^{p-1} .
$$

Finally, to find the appropriate estimate for $B_{2}$, observe that for $u \in G_{n}$,

$$
\begin{aligned}
B_{2} \leq & \left(\int_{G_{j} \backslash G_{j+1}}\left|\left(\varphi_{k}\right)^{\vee}(y-u)\right|^{r} d \mu(y)\right)^{p / r} \\
& \cdot\left(\int_{G_{j} \backslash G_{j+1}}\left(v_{\alpha}\left(x_{0}+y\right)\right)^{r /(r-p)} d \mu(y)\right)^{(r-p) / r} \\
\leq & C\left\|\left(\varphi_{k}\right)^{\vee} \chi_{G_{j} \backslash G_{j+1}}\right\|_{r}^{p} \cdot\left(m_{j}\right)^{-(\alpha+1-p / r)} \\
\leq & C\left(m_{j}\right)^{-\alpha}\left\|\left(\varphi_{k}\right)^{\vee}\right\|_{K(1 / p-1 / r, r, p)}^{p} \\
\leq & C\left(m_{j}\right)^{-\alpha}\left(m_{k}\right)^{p-1} .
\end{aligned}
$$

Therefore,

$$
B \leq C\|a\|_{\infty}^{p} \cdot\left(m_{k}\right)^{1-p}\left(m_{j}\right)^{-\alpha}\left(m_{k}\right)^{p-1}\left(m_{n}\right)^{-1} \leq C,
$$

because $a$ is a $(p, \infty)_{\alpha}$ atom. Thus we see that $\left\|\left(\varphi_{k} \hat{a}\right)^{\vee}\right\|_{p, \alpha}^{p} \leq C$.

In case supp $a \subset G_{n}$ we have $\|a\|_{\infty} \leq\left(\mu_{\alpha}\left(G_{n}\right)\right)^{-1 / p} \leq C\left(m_{n}\right)^{(\alpha+1) / p}$, and for each $k \in \mathbb{Z}$,

$$
\begin{aligned}
\left\|\left(\varphi_{k} \hat{a}\right)^{\vee}\right\|_{p, \alpha}^{p} & =\left\|\left(\varphi_{k}\right)^{\vee} * a\right\|_{p, a}^{p} \\
& =\left\|\left(\left(\varphi_{k}\right)^{\vee} * a\right) \chi_{G_{n}}\right\|_{p, \alpha}^{p}+\left\|\left(\left(\varphi_{k}\right)^{\vee} * a\right) \chi_{G \backslash G_{n}}\right\|_{p, \alpha}^{p} \\
& :=A+B .
\end{aligned}
$$


Choose $s>1$ so that $-1+p / s<\alpha$. Then, according to Lemma 4.4, $\varphi_{k} \in \mathcal{M}\left(L^{s}\right)$ and we see that

$$
\begin{aligned}
A & \leq\left(\int_{G_{n}}\left|\left(\varphi_{k}\right)^{\vee} * a(x)\right|^{s} d \mu(x)\right)^{p / s} \cdot\left(\int_{G_{n}}\left(v_{\alpha}(x)\right)^{s /(s-p)} d \mu(x)\right)^{(s-p) / s} \\
& \leq C\left\|\left(\varphi_{k}\right)^{\vee} * a\right\|_{s}^{p} \cdot\left(m_{n}\right)^{-(\alpha+1-p / s)} \\
& \leq C\|a\|_{s}^{p} \cdot\left(m_{n}\right)^{-(\alpha+1-p / s)} \leq C .
\end{aligned}
$$

Moreover, as in the first part of the proof, we have

$$
\begin{aligned}
B & \leq\|a\|_{\infty}^{p} \cdot\left(m_{k}\right)^{1-p} \int_{G_{n}} \int_{G \backslash G_{n}}\left|\left(\varphi_{k}\right)^{\vee}(y-u)\right|^{p} v_{\alpha}(y) d \mu(y) d \mu(u) \\
& \leq C\|a\|_{\infty}^{p}\left(m_{k}\right)^{1-p}\left(m_{n}\right)^{-\alpha}\left(m_{k}\right)^{p-1}\left(m_{n}\right)^{-1} \leq C .
\end{aligned}
$$

Thus, we see again that $\|\left(\left(\varphi_{k} \hat{a}\right)^{\vee} \|_{p, \alpha}^{p} \leq C\right.$. According to Remark 4.2 we may conclude that $\varphi \in \mathcal{M}\left(H_{\alpha}^{p}, L_{\alpha}^{p}\right)$.

The next theorem deals with multipliers from $H_{\alpha}^{p}$ to $H_{\alpha}^{p}$. We begin with a lemma which extends [9, Theorem 2].

LEMMA 4.6. Let $\varphi \in L^{\infty}(\Gamma)$ and $0<p \leq 1$. If

$$
\sup _{k}\left(m_{k}\right)^{1 / p-1} \sum_{j=k}^{\infty}\left\|\left(\varphi^{j}\right)^{\vee}\right\|_{K(1 / p-1 / r, r, 1)}<\infty
$$

for some $r$ with $1 \leq r<\infty$ then $\varphi \in \mathcal{M}\left(H^{s}\right)$ for $1 \leq s<\infty$.

Proof. We first prove that $\varphi \in \mathcal{M}\left(H^{1}\right)$ by showing that there exists a $C>0$ so that for all $(1, \infty)$ atoms $a$ we have $\left\|(\varphi \hat{a})^{\vee}\right\|_{H^{1}} \leq C$. We may assume that supp $a \subset G_{n}$ for some $n \in \mathbb{Z}$. Let $f=(\varphi \hat{a})^{\vee}$ and let $f^{*}=\sup _{l}\left|f * \Delta_{l}\right|$. Kitada showed in [4, Theorem 2] that

$$
\int_{G_{n}} f^{*}(x) d \mu(x) \leq C
$$

and

$$
\int_{G \backslash G_{n}} f^{*}(x) d \mu(x) \leq \sum_{j=n}^{\infty} \sum_{k=-\infty}^{n-1}\left\|\left(\varphi^{j}\right)^{\vee} \chi_{G_{k} \backslash G_{k+1}}\right\|_{1} .
$$

Applying Hölder's inequality we see that for $k<n$

$$
\begin{aligned}
\left\|\left(\varphi^{j}\right)^{\vee} \chi_{G_{k} \backslash G_{k+1}}\right\|_{1} & \leq\left\|\left(\varphi^{j}\right)^{\vee} \chi_{G_{k} \backslash G_{k+1}}\right\|_{r} \cdot\left(m_{k}\right)^{-1 / r^{\prime}} \\
& =\left(m_{k}\right)^{1 / p-1}\left(m_{k}\right)^{-(1 / p-1 / r)}\left\|\left(\varphi^{j}\right)^{\vee} \chi_{G_{k} \backslash G_{k+1}}\right\|_{r} \\
& \leq\left(m_{n}\right)^{1 / p-1}\left(m_{k}\right)^{-(1 / p-1 / r)}\left\|\left(\varphi^{j}\right)^{\vee} \chi_{G_{k} \backslash G_{k+1}}\right\|_{r} .
\end{aligned}
$$


Hence, we have

$$
\begin{aligned}
\int_{G \backslash G_{n}} f^{*}(x) d \mu(x) & \leq\left(m_{n}\right)^{1 / p-1} \sum_{j=n}^{\infty} \sum_{k=-\infty}^{n-1}\left(m_{k}\right)^{-(1 / p-1 / r)}\left\|\left(\varphi^{j}\right)^{\vee} \chi_{G_{k} \backslash G_{k+1}}\right\|_{r} \\
& \leq\left(m_{n}\right)^{1 / p-1} \sum_{j=n}^{\infty}\left\|\left(\varphi^{j}\right)^{\vee}\right\|_{K(1 / p-1 / r, r, 1)} \leq C .
\end{aligned}
$$

Therefore,

$$
\|f\|_{H^{1}}=\int_{G_{n}} f^{*}(x) d \mu(x)+\int_{G \backslash G_{n}} f^{*}(x) d \mu(x) \leq C,
$$

that is, $\varphi \in \mathcal{M}\left(H^{1}\right)$.

We now show that $\varphi \in \mathcal{M}\left(H^{s}\right)$ for $1<s<\infty$. Since $\varphi \in \mathcal{M}\left(H^{1}\right)$ there exists a $C>0$ so that for all $f \in H^{1} \cap L^{2}$ we have

$$
\|T f\|_{1}:=\left\|(\varphi \hat{f})^{\vee}\right\|_{L^{1}} \leq\left\|(\varphi \hat{f})^{\vee}\right\|_{H^{1}} \leq C\|f\|_{H^{1}},
$$

that is, $\varphi \in \mathcal{M}\left(H^{1}, L^{1}\right)$. Since $H^{1} \cap L^{2}$ is a dense subset of $H^{1}$, the operator $T$ can be extended to $H^{1}$ so that $\|T f\|_{H^{1}} \leq C\|f\|_{H^{1}}$ for all $f \in H^{1}$. This implies immediately that $T$ is of weak type $\left(H^{1}, 1\right)$ on $H^{1}$. Since $\varphi \in \mathcal{M}\left(L^{2}\right), T$ is of type $(2,2)$ on $L^{2}$. Thus, it follows from Theorem 3.22 and a standard duality argument that $T$ is of type $(s, s)$, that is, $\varphi \in$ $\mathcal{M}\left(L^{s}\right)=\mathcal{M}\left(H^{s}\right)$ for each $1<s<\infty$.

ThEOREM 4.7. Let $\varphi \in L^{\infty}(\Gamma)$ and $0<p \leq 1$. If

$$
\sup _{k}\left(m_{k}\right)^{1-p} \sum_{j=k}^{\infty}\left\|\left(\varphi^{j}\right)^{\vee}\right\|_{K(1 / p-1 / r, r, p)}^{p}<\infty
$$

for some $r$ with $1 \leq r<\infty$ then $\varphi \in \mathcal{M}\left(H_{\alpha}^{p}\right)$ for $-1+p / r<\alpha \leq 0$.

Proof. Since $0<p \leq 1$ we have

$$
\begin{aligned}
& \left(m_{k}\right)^{1-p}\left(\sum_{j=k}^{\infty}\left\|\left(\varphi^{j}\right)^{\vee}\right\|_{K(1 / p-1 / r, r, 1)}\right)^{p} \leq\left(m_{k}\right)^{1-p} \sum_{j=k}^{\infty}\left\|\left(\varphi^{j}\right)^{\vee}\right\|_{K(1 / p-1 / r, r, 1)}^{p} \\
& \leq C\left(m_{k}\right)^{1-p} \sum_{j=k}^{\infty}\left\|\left(\varphi^{j}\right)^{\vee}\right\|_{K(1 / p-1 / r, r, p)}^{p}<\infty .
\end{aligned}
$$

It follows from Lemma 4.6 that $\varphi \in \mathcal{M}\left(H^{s}\right)$ for $1 \leq s<\infty$.

To see that $\varphi \in \mathcal{M}\left(H_{\alpha}^{p}\right)$ for $-1+p / r<\alpha \leq 0$, let $a$ be a $(p, \infty)_{\alpha}$ atom with supp $a \subset I=x_{0}+G_{n}$. Take any $k \in \mathbb{Z}$ and let $f=\left(\varphi_{k} \hat{a}\right)^{\vee}=\left(\varphi_{k}\right)^{\vee} * a$ 
and let $f^{*}=\sup _{l}\left|f * \Delta_{l}\right|$. We have

$$
\begin{aligned}
& \int_{G}\left(f^{*}(x)\right)^{p} d \mu_{\alpha}(x) \\
& \quad=\int_{I}\left(f^{*}(x)\right)^{p} d \mu_{\alpha}(x)+\int_{G \backslash I}\left(f^{*}(x)\right)^{p} d \mu_{\alpha}(x):=A+B .
\end{aligned}
$$

To estimate $A$ we distinguish two cases.

(i) If $x_{0} \in G_{n}$ then for every $r \in[1, \infty)$ and each $\alpha$ with $-1+p / r<$ $\alpha \leq 0$ we have

$$
\begin{aligned}
A & \leq\left(\int_{G_{n}}\left(f^{*}(x)\right)^{r} d \mu(x)\right)^{p / r} \cdot\left(\int_{G_{n}}\left(v_{\alpha}(x)\right)^{r /(r-p)} d \mu(x)\right)^{(r-p) / r} \\
& \leq C\|a\|_{H^{r}}^{p} \cdot\left(m_{n}\right)^{-(\alpha+1-p / r)} \leq C,
\end{aligned}
$$

where the second inequality is obtained by observing that $\varphi \in \mathcal{M}\left(H^{r}\right)$.

(ii) If $x_{0} \notin G_{n}$ then $x_{0} \in G_{l} \backslash G_{l+1}$ for some $l<n$ and $I \subset G_{l} \backslash G_{l+1}$. With $r$ and $\alpha$ as in (i) we have

$$
\begin{aligned}
A & \leq\left(\int_{I}\left(f^{*}(x)\right)^{r} d \mu(x)\right)^{p / r} \cdot\left(\int_{I}\left(v_{\alpha}(x)\right)^{r /(r-p)} d \mu(x)\right)^{(r-p) / r} \\
& \leq C\|a\|_{H^{r}}^{p} \cdot\left(m_{l}\right)^{-\alpha}\left(m_{n}\right)^{-(1-p / r)} \leq C .
\end{aligned}
$$

To find the appropriate estimate for $B$ we closely follow Kitada's proof of [5, Theorem 2]. If we set $\psi(\gamma)=\overline{\gamma\left(x_{0}\right)} \varphi(\gamma), \psi^{l}=\psi \chi_{\Gamma_{l+1} \backslash \Gamma_{l}}$ and $b(x)=$ $a\left(x+x_{0}\right)$, then Kitada showed that

$$
f^{*}(x) \leq \sum_{j=n}^{\infty}\left|\left(\psi^{j}\right)^{\vee} * b(x)\right| .
$$

Therefore,

$$
\begin{aligned}
B & =\int_{G \backslash I}\left(f^{*}(x)\right)^{p} d \mu_{\alpha}(x) \\
& \leq \sum_{j=n}^{\infty} \sum_{i=-\infty}^{n-1} \int_{J_{i}}\left|\left(\psi^{j}\right)^{\vee} * b(x)\right|^{p} d \mu_{\alpha}(x),
\end{aligned}
$$

where $J_{i}=I_{i} \backslash I_{i+1}$ and $I_{i}=x_{0}+G_{i}$. For each of the integrals in this sum Kitada showed that

$$
\begin{aligned}
B_{i j} & :=\int_{J_{i}}\left|\left(\psi^{j}\right)^{\vee} * b(x)\right|^{p} d \mu_{\alpha}(x) \\
& =\int_{J_{i}}\left|\left(\psi^{j}\right)^{\vee} \chi_{J_{i}} * b(x)\right|^{p} d \mu_{\alpha}(x) .
\end{aligned}
$$


Consequently, applying [7, Lemma 1(b)] to obtain the third inequality, we see that

$$
\begin{aligned}
B_{i j} \leq & \left(\int_{J_{i}}\left|\left(\psi^{j}\right)^{\vee} \chi_{J_{i}} * b(x)\right|^{r} d \mu(x)\right)^{p / r} \\
& \cdot\left(\int_{J_{i}}\left(v_{\alpha}(x)\right)^{r /(r-p)} d \mu(x)\right)^{(r-p) / r} \\
\leq & \|b\|_{1}^{p}\left\|\left(\psi^{j}\right)^{\vee} \chi_{J_{i}}\right\|_{r}^{p} \cdot\left(\mu_{\alpha r /(r-p)}\left(I_{i}\right)\right)^{(r-p) / r} \\
\leq & \|a\|_{1}^{p}\left\|\left(\varphi^{j}\right)^{\vee} \chi_{G_{i} \backslash G_{i+1}}\right\|_{r}^{p} \cdot C\left(\left(m_{i}\right)^{-1} \inf \left\{v_{\alpha r /(r-p)}(y): y \in I_{i} \backslash\{0\}\right\}\right)^{(r-p) / r} \\
\leq & C\|a\|_{1}^{p}\left\|\left(\varphi^{j}\right)^{\vee} \chi_{G_{i} \backslash G_{i+1}}\right\|_{r}^{p} \\
& \cdot\left(m_{i}\right)^{(p-r) / r}\left(\inf \left\{v_{\alpha r / r(r-p)}(y): y \in I_{i} \backslash\{0\}\right\}\right)^{(r-p) / r}
\end{aligned}
$$

(a) If $I_{n}=I=G_{n}$ we have

$$
B_{i j} \leq C\left(m_{n}\right)^{\alpha+1-p}\left\|\left(\varphi^{j}\right)^{\vee} \chi_{G_{i} \backslash G_{i+1}}\right\|_{r}^{p} \cdot\left(m_{i}\right)^{(p-r) / r}\left(m_{n}\right)^{-\alpha} .
$$

(b) If $I_{n} \subset G_{l} \backslash G_{l+1}$ for some $l<n$ we have

$$
B_{i j} \leq C\left(m_{l}\right)^{\alpha}\left(m_{n}\right)^{1-p}\left\|\left(\varphi^{j}\right)^{\vee} \chi_{G_{i} \backslash G_{i+1}}\right\|_{r}^{p} \cdot\left(m_{i}\right)^{(p-r) / r}\left(m_{l}\right)^{-\alpha} .
$$

Thus, in both cases we see that

$$
\begin{aligned}
B & \leq C\left(m_{n}\right)^{1-p} \sum_{j=n}^{\infty} \sum_{i=-\infty}^{n-1}\left(\left(m_{i}\right)^{1 / r-1 / p}\left\|\left(\varphi^{j}\right)^{\vee} \chi_{G_{i} \backslash G_{i+1}}\right\|_{r}\right)^{p} \\
& \leq C\left(m_{n}\right)^{1-p} \sum_{j=n}^{\infty}\left\|\left(\varphi^{j}\right)^{\vee}\right\|_{K(1 / p-1 / r, r, p)}^{p} \leq C .
\end{aligned}
$$

Thus, $\left\|f^{*}\right\|_{p, \alpha}=\|f\|_{H_{\alpha}^{p}} \leq C$ and this implies that $\varphi \in \mathcal{M}\left(H_{\alpha}^{p}\right)$.

For $0<p<1$ we have the following corollary.

Corollary 4.8. Let $\varphi \in L^{\infty}(\Gamma)$ and $0<p<1$. If

$$
\sup _{k}\left(m_{k}\right)^{1 / p-1}\left\|\left(\varphi^{k}\right)^{\vee}\right\|_{K(1 / p-1 / r, r, p)}<\infty
$$

for some $r$ with $1 \leq r<\infty$ then $\varphi \in \mathcal{M}\left(H_{\alpha}^{p}\right)$ for $-1+p / r<\alpha \leq 0$.

Proof. For $0<p<1$ we have

$$
\sum_{j=k}^{\infty} \|\left\langle( \varphi ^ { j } ) ^ { v } \left\langle\left.\right|_{K(1 / p-1 / r, r, p)} ^{p} \leq \mathrm{C} \sum_{j=k}^{\infty}\left(m_{j}\right)^{0-1} \leq \mathrm{C}\left(m_{k}\right)^{0-1} .\right.\right.
$$


The result follows immediately from Theorem 4.7.

We now show that Corollary 4.8 is sharp in a certain sense. The example we use to prove the sharpness result is a variation of the example used in [9] to prove that certain results of Kitada for $H^{p}$ multipliers, $0<p<1$, were best possible.

THEOREM 4.9. Let $0<p<1$ and $1 \leq r<\infty$. There exists $a \varphi \in L^{\infty}(\Gamma)$ so that

(i) $\sup _{k}\left(m_{k}\right)^{1 / p-1}\left\|\left(\varphi^{k}\right)^{\vee}\right\|_{K(1 / p-1 / r, r, q)}<\infty$ for every $q>p$;

(ii) $\varphi \in \mathcal{M}\left(H_{\alpha}^{q}\right)$ for all $q$ with $p<q<1$ and $\alpha$ with $-1+q / r<\alpha \leq 0$;

(iii) $\varphi \notin \mathcal{M}\left(H_{\alpha}^{p}\right)$ for any $\alpha$ with $-1<\alpha \leq 0$.

Proof. Choose $\gamma_{1} \in \Gamma_{1} \backslash \Gamma_{0}$ and define $f: G \rightarrow \mathbb{C}$ by

$$
f(x)=\sum_{k=-\infty}^{-1}\left(\frac{m_{k}}{|k|}\right)^{1 / p} \gamma_{1}(x) \chi_{G_{k} \backslash G_{k+1}}(x) .
$$

Then $f \in L^{1}(G)$ and for every $r \geq p$ we have

$$
\begin{aligned}
\|f\|_{K(1 / p-1 / r, r, q)}^{q} & =\sum_{k=-\infty}^{\infty}\left(m_{k}\right)^{-(1 / p-1 / r) q}\left\|f \chi_{G_{k} \backslash G_{k+1}}\right\|_{r}^{q} \\
& \cong \sum_{k=-\infty}^{-1}\left(\frac{1}{|k|}\right)^{q / p}<\infty \Leftrightarrow q>p .
\end{aligned}
$$

Moreover, if $q>p$ then

$$
\begin{aligned}
\|f\|_{K(1 / q-1 / r, r, q)}^{q} & =\sum_{k=-\infty}^{\infty}\left(m_{k}\right)^{-(1 / q-1 / r) q}\left\|f \chi_{G_{k} \backslash G_{k+1}}\right\|_{r}^{q} \\
& =\sum_{k=-\infty}^{-1}\left(m_{k}\right)^{-1+q / r}\left(\frac{m_{k}}{|k|}\right)^{q / p}\left(\mu\left(G_{k} \backslash G_{k+1}\right)\right)^{q / r} \\
& \leq C \sum_{k=-\infty}^{-1}\left(\frac{1}{|k|}\right)^{q / p}\left(m_{k}\right)^{-1+q / p}<\infty
\end{aligned}
$$

Also,

$$
\hat{f}(\gamma)=\sum_{k=-\infty}^{-1}\left(\frac{m_{k}}{|k|}\right)^{1 / p}\left(\hat{\chi}_{G_{k}}-\hat{\chi}_{G_{k+1}}\right)\left(\gamma-\gamma_{1}\right),
$$

with $\hat{\chi}_{G_{k}}=m_{k} \chi_{\Gamma_{k}}$. Thus supp $\hat{f} \subset \gamma_{1}+\Gamma_{0} \subset \Gamma_{1} \backslash \Gamma_{0}$. Let $\varphi=\hat{f}$. Then $\varphi \in L^{\infty}(\Gamma)$. Moreover, $\varphi^{k}=0$ for $k \neq 1$ and $\varphi^{k}=\varphi$ for $k=1$, so that $\left(\varphi^{1}\right)^{\vee}=f$ and $\left(\varphi^{k}\right)^{\vee}=0$ for $k \neq 1$. Therefore $\varphi$ satisfies (i) and, 
according to Corollary 4.8, $\varphi$ satisfies (ii). To see that $\varphi$ satisfies (iii), choose for every $i<0$ an $x_{i} \in G_{i} \backslash G_{i+1}$ and define, for $-1<\alpha \leq 0$, functions $g_{i}: G \rightarrow \mathbb{C}$ by

$$
g_{i}(x)=\left(m_{i}\right)^{\alpha / p}\left(m_{1} \chi_{x_{i}+G_{1}}-m_{0} \chi_{x_{i}+G_{0}}\right)(x) .
$$

Then $g_{i}$ is a multiple of a $(p, \infty)_{\alpha}$ atom and $\left\|g_{i}\right\|_{H_{\alpha}^{p}} \leq m_{1}$. Moreover,

$$
\hat{g}_{i}(\gamma)=\left(m_{i}\right)^{\alpha / p} \overline{\gamma\left(x_{i}\right)}\left(\chi_{\Gamma_{1}}-\chi_{\Gamma_{\mathbf{0}}}\right)(\gamma),
$$

so supp $\hat{g}_{i} \subset \Gamma_{1} \backslash \Gamma_{0}$.

Furthermore, if we define $h_{i}: G \rightarrow \mathbb{C}$ by

$$
h_{i}(x)=\left(m_{i}\right)^{\alpha / p} \sum_{k=-\infty}^{-1}\left(\frac{m_{k}}{|k|}\right)^{1 / p} \gamma_{1}\left(x-x_{i}\right) \chi_{G_{k} \backslash G_{k+1}}\left(x-x_{i}\right)
$$

then $h_{i} \in L^{1}(G)$, and a straightforward computation shows that $\hat{h}_{i}=\varphi \hat{g}_{i}$, that is, $h_{i}=\left(\varphi \hat{g}_{i}\right)^{\vee}$. Furthermore, we have

$$
\left\|h_{i}\right\|_{p, \alpha}^{p}=\int_{G}\left|h_{i}(x)\right|^{p} d \mu_{\alpha}(x) \geq C \sum_{k=i}^{-1}|k|^{-1}
$$

so $\lim _{i \rightarrow-\infty}\left\|h_{i}\right\|_{p, \alpha}=\infty$. Since each $h_{i} \in L^{1}(G)$ we have

$$
\left\|h_{i}\right\|_{H_{\alpha}^{p}}=\left\|h_{i}^{*}\right\|_{p, \alpha} \geq\left\|h_{i}\right\|_{p, \alpha} \text {, }
$$

so

$$
\lim _{i \rightarrow-\infty}\left\|\left(\varphi \hat{g}_{i}\right)^{\vee}\right\|_{H_{\alpha}^{p}}=\lim _{i \rightarrow-\infty}\left\|h_{i}\right\|_{H_{\alpha}^{p}}=\lim _{i \rightarrow-\infty}\left\|h_{i}^{*}\right\|_{p, \alpha}=\infty
$$

and this implies that $\varphi \notin \mathcal{M}\left(H_{\alpha}^{p}\right)$.

In his most recent paper on multipliers on $H^{p}(G)$ spaces [6], Kitada proved a multiplier result for Hardy spaces on locally compact Vilenkin groups in which his assumptions are the natural analogue for $G$ of the usual Hörmander condition for multipliers for function spaces on $\mathbb{R}^{n}$. Before stating Kitada's main result we first repeat a definition given in [6].

Definition 4.10. Let $\varphi \in L^{\infty}(\Gamma)$. For $\lambda>0$ and $j \in \mathbb{Z}$ let $D^{\lambda} \varphi^{j}$ be defined by

$$
D^{\lambda} \varphi^{j}=\left(|x|^{\lambda}\left(\varphi^{j}\right)^{\vee}(x)\right)^{\wedge}
$$

We say that $\varphi \in M(s, \lambda)$, where $1 \leq s \leq \infty$, if

$$
B(\varphi, s, \lambda):=\|\varphi\|_{\infty}+\sup _{j}\left(m_{j}\right)^{\lambda-1 / s}\left\|D^{\lambda} \varphi^{j}\right\|_{s}<\infty .
$$

In [6, Theorem 2] Kitada proved the following, which is the analogue for $G$ of [12, Theorem (4.11)]. 
THEOREM K. Let $0<p \leq 1$ and $1 \leq s<\infty$. If $\varphi \in M(s, \lambda)$ for $\lambda>1 / p-1 / \max \left(2, s^{\prime}\right)$ then $\varphi \in \mathcal{M}\left(H^{p}\right)$.

We conclude this paper by extending Theorem $\mathrm{K}$ to power-weighted Hardy spaces. Our proof depends on Corollary 4.8 and is somewhat different form Kitada's proof of Theorem $\mathrm{K}$. We first establish a simple lemma.

LeMma 4.11. Let $\varphi \in L^{\infty}(\Gamma)$, let $0<p \leq 1$ and $1 \leq r<\infty$. If

$$
\sup _{k}\left(m_{k}\right)^{1 / p-1+\varepsilon}\left\|\left(\varphi^{k}\right)^{\vee}\right\|_{K(1 / p-1 / r+\varepsilon, r, \infty)}<\infty \quad \text { for some } \varepsilon>0,
$$

then

$$
\sup _{k}\left(m_{k}\right)^{1 / p-1}\left\|\left(\varphi^{k}\right)^{\vee}\right\|_{K(1 / p-1 / r, r, p)}<\infty .
$$

Proof. We have

$$
\begin{aligned}
\left\|\left(\varphi^{k}\right)^{\vee}\right\|_{K(1 / p-1 / r, r, p)}^{p}= & \sum_{i=-\infty}^{k}\left(m_{i}\right)^{-1+p / r}\left\|\left(\varphi^{k}\right)^{\vee} \chi_{G_{i} \backslash G_{i+1}}\right\|_{r}^{p} \\
& +\sum_{i=k+1}^{\infty}\left(m_{i}\right)^{-1+p / r}\left\|\left(\varphi^{k}\right)^{\vee} \chi_{G_{i} \backslash G_{i+1}}\right\|_{r}^{p} \\
:= & A+B .
\end{aligned}
$$

Assumption (4.12) implies that

$$
\begin{aligned}
A & \leq C \sum_{i=-\infty}^{k}\left(m_{i}\right)^{-1+p / r}\left(m_{i}\right)^{1-p / r+\varepsilon p}\left(m_{k}\right)^{-1+p-\varepsilon p} \\
& \leq C\left(m_{k}\right)^{-1+p-\varepsilon p} \sum_{i=-\infty}^{k}\left(m_{i}\right)^{\varepsilon p} \leq C\left(m_{k}\right)^{-1+p}
\end{aligned}
$$

since $\varepsilon p>0$.

To estimate $B$ first observe that

$$
\left\|\left(\varphi^{k}\right)^{\vee}\right\|_{\infty} \leq\left\|\varphi^{k}\right\|_{1} \leq\left\|\varphi^{k}\right\|_{\infty} \cdot \lambda\left(\Gamma_{k+1} \backslash \Gamma_{k}\right) \leq C\|\varphi\|_{\infty} \cdot m_{k} .
$$

Therefore,

$$
\begin{aligned}
B & \leq C \sum_{i=k+1}^{\infty}\left(m_{i}\right)^{-1+p / r}\left(m_{k}\right)^{p}\left(m_{i}\right)^{-p / r} \\
& =C\left(m_{k}\right)^{p} \sum_{i=k+1}^{\infty}\left(m_{i}\right)^{-1} \leq C\left(m_{k}\right)^{p-1} .
\end{aligned}
$$

From the inequalities for $A$ and $B$ we immediately obtain (4.13). 
Corollary 4.14. Let $\varphi \in L^{\infty}(\Gamma)$, let $0<p \leq 1$ and $1 \leq r<\infty$. If $\varphi$ satisfies (4.12) then $\varphi \in \mathcal{M}\left(H_{\alpha}^{p}\right)$ for $-1+p / r<\alpha \leq 0$.

Proof. For $p=1$ this is [5, Theorem 2]. For $0<p<1$ we apply Lemma 4.11 and Corollary 4.8.

TheOREM 4.15. Let $\varphi \in L^{\infty}(\Gamma)$, let $0<p \leq 1,1<r \leq \infty$ and $\lambda>1 / p-$ $1 / \max \left(2, r^{\prime}\right)$. If $\varphi \in M(r, \lambda)$ then $\varphi \in \mathcal{M}\left(H_{\alpha}^{p}\right)$ for $-1+p / \min \left(2, r^{\prime}\right)<$ $\alpha \leq 0$.

Proof. We first assume that $1<r \leq 2$ so that $\max \left(2, r^{\prime}\right)=r^{\prime}$. Since $\lambda>1 / p-1 / r^{\prime}$, there exists an $\varepsilon>0$ so that $\lambda=1 / p-1 / r^{\prime}+\varepsilon$. Now we consider

$$
\begin{gathered}
\left\|\left(\varphi^{j}\right)^{\vee}\right\|_{K\left(1 / p-1 / r^{\prime}+\varepsilon, r^{\prime}, \infty\right)}=\left\|\left(\varphi^{j}\right)^{\vee}\right\|_{K\left(\lambda, r^{\prime}, \infty\right)} \leq\left\|\left(\varphi^{j}\right)^{\vee}\right\|_{K\left(\lambda, r^{\prime}, r^{\prime}\right)} \\
\quad=\left\|\left(\varphi^{j}\right)^{\vee}\right\|_{r^{\prime}, \lambda r^{\prime}}=\left\|\left.x\right|^{\lambda}\left(\varphi^{j}\right)^{\vee}\right\|_{r^{\prime}}=\left\|\left(D^{\lambda} \varphi^{j}\right)^{\vee}\right\|_{r^{\prime}} \leq\left\|\left(D^{\lambda} \varphi^{j}\right)\right\|_{r^{\prime}} .
\end{gathered}
$$

Thus, if $\varphi \in M(r, \lambda)$ then $\varphi$ satisfies inequality (4.12), and Corollary 4.14 implies that $\varphi \in \mathcal{M}\left(H_{\alpha}^{p}\right)$ for $-1+p / r^{\prime}<\alpha \leq 0$.

If $2<r \leq \infty$ then $\max \left(2, r^{\prime}\right)=2$. In this case there exists an $\varepsilon>0$ such that $\lambda=1 / p-1 / 2+\varepsilon$ and we have

$$
\left\|\left(\varphi^{j}\right)^{\vee}\right\|_{K(1 / p-1 / 2+\varepsilon, 2, \infty)}=\left\|\left(\varphi^{j}\right)^{\vee}\right\|_{K(\lambda, 2, \infty)} \leq\left\|\left(\varphi^{j}\right)^{\vee}\right\|_{K(\lambda, 2,2)} \leq\left\|D^{\lambda} \varphi^{j}\right\|_{2} .
$$

An application of [6, Proposition 2] to obtain the third inequality, shows that

$$
\begin{aligned}
& \sup _{j}\left(m_{j}\right)^{1 / p-1+\varepsilon}\left\|\left(\varphi^{j}\right)^{\vee}\right\|_{K(1 / p-1 / 2+\varepsilon, 2, \infty)} \\
& \quad \leq \sup _{j}\left(m_{j}\right)^{\lambda-1 / 2}\left\|D^{\lambda} \varphi^{j}\right\|_{2} \leq B(\varphi, 2, \lambda) \\
& \leq C B(\varphi, r, \lambda)<\infty
\end{aligned}
$$

and the conclusion of the theorem follows again from Corollary 4.14. This completes the proof of Theorem 4.15.

Remark. Professor Kitada informed the authors that he obtained independently essentially the same result as our Theorem 4.15 .

\section{References}

[1] R. Coifman and G. Weiss, 'Extensions of Hardy spaces and their use in analysis', Bull. Amer. Math. Soc. 83 (1977), 569-645.

[2] R. E. Edwards and G. I. Gaudry, Littlewood-Paley and multiplier theory, (SpringerVerlag, Berlin, 1977). 
[3] J. Garcia-Cuerva and J. L. Rubio de Francia, Weighted norm inequalities and related topics, (North-Holland Mathematics Studies 116, North-Holland, Amsterdam, 1985).

[4] T. Kitada, ' $H^{p}$-multiplier theorems on certain totally disconnected groups', Sci. Rep. Hirosaki Univ. 34 (1987), 1-7.

[5] T. Kitada, 'Multipliers on weighted Hardy spaces over certain totally disconnected groups', Internat. J. Math. Math. Sci. 11 (1988), 665-675.

[6] T. Kitada, ' $H^{p}$ multiplier theorem on certain totally disconnected groups, II', preprint.

[7] C. W. Onneweer, 'Multipliers on weighted $L^{p}$-spaces over certain totally disconnected groups', Trans. Amer. Math. Soc. 288 (1985), 347-357.

[8] C. W. Onneweer and T. S. Quek, 'Multipliers on weighted $L^{p}$-spaces over locally compact Vilenkin groups', Proc. Amer. Math. Soc. 105 (1989), 622-631.

[9] C. W. Onneweer and T. S. Quek, ' $H$ ' multiplier results on locally compact Vilenkin groups', Quart. J. Math. Oxford Ser. (2) 40 (1989), 313-323.

[10] M. H. Taibleson, 'Harmonic analysis on $n$-dimensional vector spaces over local fields, III. Multipliers', Math. Ann. 187 (1970), 259-271.

[11] M. H. Taibleson, Fourier analysis on local fields, (Mathematical Notes 15, Princeton University Press, 1975).

[12] M. H. Taibleson and G. Weiss, The molecular characterization of certain Hardy spaces, (Astérisque 77, pp. 68-149, 1980).

[13] N. Ya. Vilenkin, On a class of complete orthonormal systems, (Amer. Math. Soc. Transl. (2) 28 , pp. 1-35, 1963).

Department of Mathematics and Statistics

University of New Mexico Albuquerque, New Mexico 87131 U.S.A.
Department of Mathematics National University of Singapore Singapore 0511 Republic of Singapore 